\title{
Modeling Instruments in Managerial Decision-Making: Actual Problems and Trends of Development
}

\author{
Ivan N. Drogobetsky 1[ORCID 0000-0002-9982-0361], \\ Marat F. Gumerov 2*[ORCID 0000-0002-6886-0192]
}

\author{
${ }^{1}$ Financial University under the Government of Russian Federation, Moscow, Russia \\ ${ }^{2}$ Institute of Additional Professional Education for Social Workers, Moscow, Russia \\ maratushka85@gmail.com
}

\begin{abstract}
The object of the present article are the new approaches to decision-making in management of social and economic systems. These new approaches have an aim to be more relevant to characteristics of modern economic life, such as volatility and high speed of metamorphosis. In the present exploration we expect to provide this relevance owing to three components. The first one is system economy: each manager's decision should take in account that economic system managed by the manager includes sub-systems of four types: environment, object, process and project. The second component is the connections between decision of a manager and basic functions executed by the manager in an economic system: producing, administrating, entrepreneurship and integration. And the third component which connects the previous two is a phenomenological modeling. It's being used for a long period already in nature studying and technics for designing production circumstances of particular materials. And then integrating such models forms a base for creating model which describes long-term laws of the relative materials' behavior. We offer to accept this experience to arena provided with management of economic systems. In the similar way, here phenomenological models are the base of managerial decisions for particular situations which are accompanied by lack of information, but demand making a decision in the shortest period.
\end{abstract}

Keywords: management of economic systems, managerial decision-making, phenomenological modeling

\section{INTRODUCTION}

Decision making in management is characterized as a rather specific area of activity. Because, on the one hand, it has been developing over a long period, and by now a solid base of definitions, models and tools has already been accumulated in its theory. But, on the other hand, the current circumstances of socio-economic life establish certain restrictions for the use of traditional methods of making managerial decisions. Generally, three kinds of such limits can be pointed out:

1) traditional methods are focused on the longterm stability of the controlled economic system and its external environment, but modern economic life is characterized by great instability, even in short-term periods;
2) most of the situations that develop today in the functioning of the economic system and require managerial decisions are characterized by a high degree of originality; this means that there were no precedents for such situations in the past; and for this reason, there is always a great lack of information and knowledge about the factors that determine the development of these situations;

3) time periods for decision-making in modern managerial practice are extremely limited.

All the characteristics described here allow us to conclude that at present it is necessary to talk about a new problem field in the theory and practice of management: making urgent decisions for nonstandard tasks. This is the object of this study. 
The main problem in this area is indicated as follows: there are no modeling tools that can support urgent decision-making for non-standard tasks, and now in practice it is based only on unformulated thought processes (such as intuition and heuristics).

Traditionally, the economic and management disciplines never had their own model apparatus, and they borrowed it from the natural sciences. So, due to the inability of the economy and management itself to offer any points for building knowledge in the urgent decision-making of non-standard problems, we turned our attention to natural science. And it turned out that this field contains a kind of model that is designed just for quick decisionmaking in situations where there is ignorance of their mechanisms. These are phenomenological models that, when studying natural and technical systems, describe the definitions between the increments of a pair of indicators. It is important that in this pair one of the indicators characterizes the internal influence, and the second - the reaction of the modeled system to this influence. And at the same time, the phenomenological model does not aim at a detailed description of the mechanism of this reaction. For this reason, the creation of this type of model does not require a lot of time, so urgent decisions are the area of their use [1]. At the first stage of this study, we formulated a primary hypothesis about the need to transfer the positive experience of natural science research to the management of economic systems and the use of phenomenological models as a basis for making urgent decisions on non-standard problems. Thus, the main goal of the study is concretized: the creation of archetypal methodological foundations for the use of phenomenological models when making urgent decisions on non-standard problems of managing economic systems.

Concretization led to a more precisely limited field of search, supplied with scientific works, the authors of which are trying to find the place of phenomenology in the theory and practice of management in economic systems. The conclusion of this search is that in all essays equipped with a research problem and published to date, their authors pay attention only to the general phenomenological approach as a tool for studying economic and managerial processes.

The first attempts to solve this problem were made in the 1980 s by representatives of the Siberian scientific school of R.G. Khlebopros, A. Gorban. Since then, they have published a number of essays, the last of which are devoted to the definition of laws that determine the development of the national economy in the context of structural movements [2] and the emergence of an intellectual economy [3]. Later, the results of studies were published, the authors of which pay attention to more particular aspects. These are the results of two research teams. The first one represents the Crimean Federal University, the results of which were obtained using a phenomenological approach in the study of the economic components of oil production [4]. The Department of Accounting and Analysis of the Financial University investigates the problems associated with the phenomenology of the analysis of the financial stability of commercial companies [5].

The common feature of all the results described is that they have only a qualitative expression. And none of these approaches contains quantitative models based on phenomenological theory and acceptable for making managerial decisions. So, in this study, we are trying to cover this empty field and create the basic elements of a methodology using phenomenological models for making urgent decisions in management. This main research task determines the logic and structure of this article. In the chapter "Materials and Methods" we adapt the methods of phenomenological modeling to the peculiarities of economic systems as objects of management. Then, the Results chapter presents the basics of our original methodology. In the Discussion section, we analyze the merits and demerits of these new methodological frameworks. And the last chapter, "Conclusion", presents some promising uses for this new methodology.

\section{MATERIALS AND METHODS}

The main part of this study is made up of models based on the phenomenological theory of learning. We formulated its main ideas at the beginning of the $20^{\text {th }}$ century by German thinker E. Husserl, who formulated the main credo "Back to things as to themselves!". This means that the study of a certain system should be based on facts (or a phenomenon, in Husserl's terminology) that take place in the system and are directly accepted by the consciousness of the subject studying it. And this studying subject must rely only on these facts and free his consciousness from all a priori established ideas about the object. This is a general way of gaining knowledge in the paradigm of the phenomenological approach.

This knowledge creates the basis for further movement towards a quantitative phenomenological 
model. For this, in the total volume of the accumulated phenomenon, it is necessary to single out the part characterized by quantitative indicators. Then these indicators are divided into two groups: the first is the experience of internal impact on the system, the second is the system's response to this impact. A quantitative description of the relationship between the indicators of these two groups is further used to answer the question: how should a subject influence the system in management processes?

\section{RESULTS}

According to the principles described in the previous chapter, present exploration began with deep analysis of phenomenological characteristics of managerial decision-making as branch of human activity. Detailed results of this stage were presented by the authors in particular monographies [6, 7]. Here we draw your attention only to the general conclusion that there are three historically formed sections in the theory of decision-making, each of which has its own view of the foundations of this activity. This foundation may include:

1) algorithms of operation's analysis;

2) behavioral factors;

3) system approach.

But, despite different opinions about the factors influencing decision-making, these three branches follow the common idea that this process has primarily informational and cognitive essence. Each act of decision-making is always accompanied by creating new knowledge and information on a base of some initial quantity of them. Further this means an increase in the amount of knowledge and information in a single system that unites the manager themself and the resources that they manage. One of the basic laws of the development of all types of systems says that an increase in the amount of information leads to an increase in orderliness (law of informativity and orderliness by Norbert Viner [8]).

Due to the fundamental nature of this process, it can be used when making decisions on cases, the features and laws of which are not clear or generally unknown. This means that the manager making a decision in such cases must initially use the idea that this decision changes the measure of order in the controlled system. In the future, he changes the connections between the internal elements of the system and between the system as a whole and its internal environment.
This idea gives a start to the methodological principles that algorithmize the adoption of urgent decisions for non-standard management tasks. The following basic definitions are used here:

- Economic system is a complex unity of elements controlled by a body (individual or collective) that makes the current decision, and this system constantly exchanges resources with its internal environment;

- Economic mega-system is a holistic symbiotic structure that includes an economic system and elements of its internal environment that are associated with current management decisions;

- Managerial decision is an action that, first of all, leads to an increase in the amount of information in a megasystem, and this increase in the future leads to a change in its measure of order and all processes of resource exchange between its elements.

Decision making begins with the decomposition of elements in an economic mega-system. Here it is necessary to use the basic tools for systematizing the facts accumulated by the subject's thinking in each cognitive process. This is space and time. The use of these two concepts in the management of economic systems is best represented in the approach of G.B. Kleiner. According to him, differences in space and time give 4 types of subsystems in each economic system. These types are environmental (unlimited both in space and time), objection (limited in space and unlimited in time), procedural (unlimited in space and limited in time) and projective (limited both in space and time) [9].

Here we adapt this approach for use in nonstandard tasks, where the decision-maker does not have sufficient knowledge about the spatial and temporal constraints of elements in the economic mega-system. We propose to overcome this difficulty by using the original idea of this study that decision making is, first of all, an information process. And according to this idea, the elements of the economic mega-system are divided into 4 subsystems with different roles of information about them. In particular, the objection subsystem includes elements, information about which is used not only for the present decision, but has already been used for similar previous decisions. And this information acts on them directly on the principle of action over short distances. Elements, information about which is also used for many similar decisions, but operates on the principle of long distance, are combined into 
an environmental subsystem. Thus, this subsystem influences decision making indirectly through the objection subsystem.

In addition, there are elements, information about which directly affects only the current solution - they form a projective subsystem. And the process subsystem affects only one decision, but indirectly through the projective subsystem.

The next initial idea of this study is that a managerial decision is an act that remakes the exchange of resources between elements of an economic mega-system. And these processes, like the elements, also need to be divided into groups. This division is intended for use in management tasks, so links with management functions are taken into account here. The only management concept that explores this factor belongs to I. Adizes, who identifies 4 main management functions.

The production function $(\mathrm{P})$ makes the economic system integral and its individual elements are able to provide each other with resources initially in the short term. The administration function (A) allows them to receive resources from each other in the same period. The functions of entrepreneurship (E) and integration (I) provide the same opportunities in the long run [10].

In the study, we use the classification of these 4 elementary acts of resource exchange and denote them by letters in accordance with the related management functions. As a result, the economic megasystem is presented as a unity of 4 subsystems, in which 4 types of resource exchange processes take place. In total, at the regional moment, the megasystem is characterized by 16 indicators of resource exchange. A managerial decision is an action that changes all 4 resource exchange processes and brings their indicators in 4 subsystems to new values. Figure 1 shows a general outline of this metamorphosis.

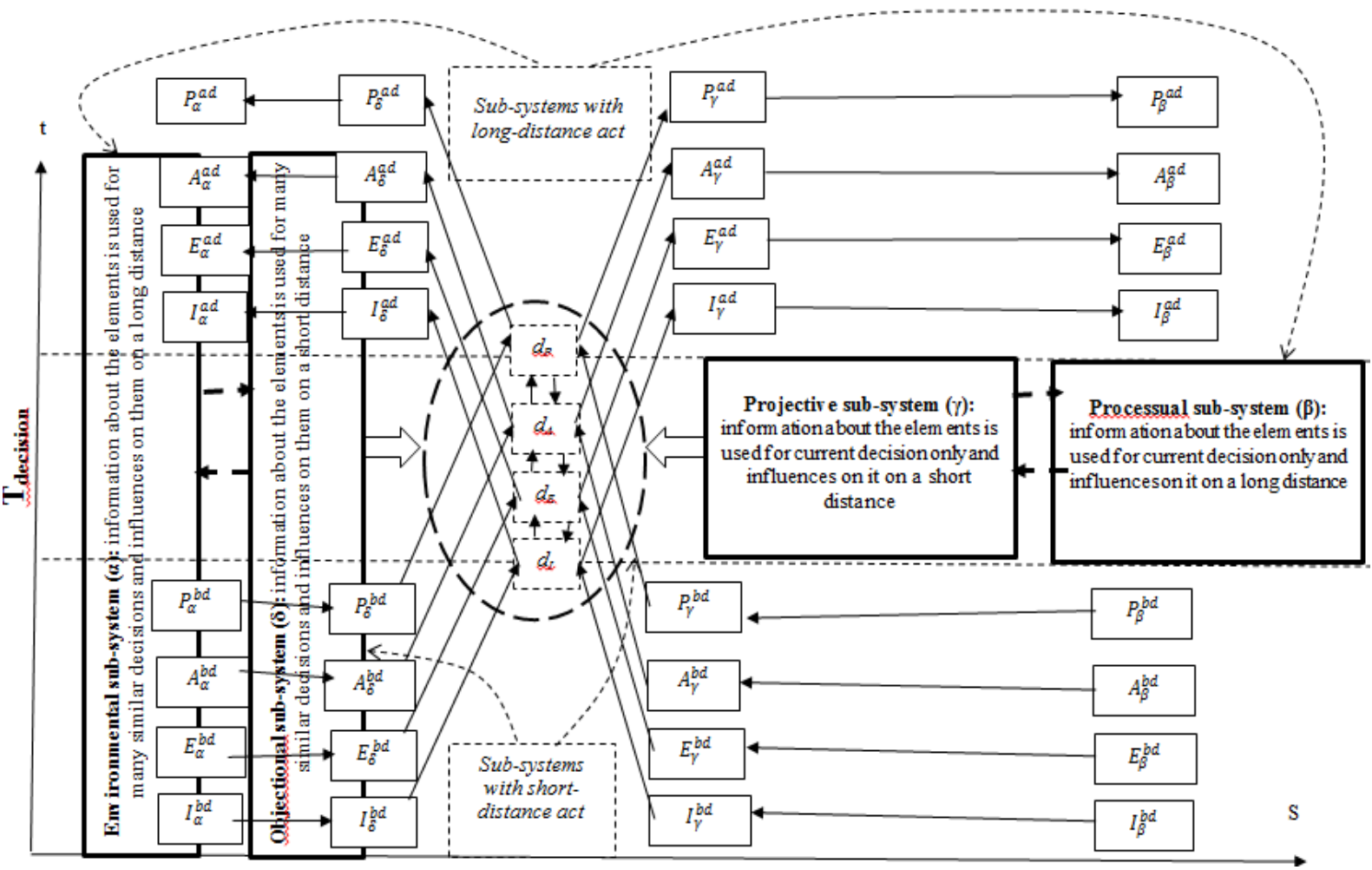

Figure 1. General scheme of decomposition of the economic mega-system when making additional decisions on emergency problems

Source: Compiled by the authors

The relationships in this figure are also formalized in the form of four equations, each of which refers to a specific resource exchange process.

In Figure 1, resource indicators of subsystems before and after a decision are shown as independent.
This illustrates a situation that is very typical for non-standard tasks: the manager does not have enough information about the definitions between these indicators, so phenomenological modeling is generally abstracted from them. 
Because, according to the basic principles, such modeling works only with the facts directly accepted by the studied subject (this role is played here by the decision-maker). At the same time, the parameters of the solution, denoted as $d$, are the only part of the mega-system built by the manager's thinking, and he also builds a definition between them. Therefore, in Figure 1, the solution parameters are shown as related. Formally, this is expressed by accepting each $d$-parameter as a mathematical function of the other three parameters of this type.

And thanks to this expression, the separated equations (1) are combined into a single mathematical system with a single solution (4 equations with 4 unknown parameters) (2).

$$
\begin{gathered}
f_{P}\left(P_{\alpha}^{b d}, P_{\delta}^{b d}, P_{\gamma}^{b d}, P_{\beta}^{b d}, P_{\alpha}^{a d}, P_{\delta}^{a d}, P_{\gamma}^{a d}, P_{\beta}^{a d}, d_{P}\right)=0 \\
f_{A}\left(A_{\alpha}^{b d}, A_{\delta}^{b d}, A_{\gamma}^{b d}, A_{\beta}^{b d}, A_{\alpha}^{a d}, A_{\delta}^{a d}, A_{\gamma}^{a d}, A_{\beta}^{a d}, d_{A}\right)=0 \\
f_{E}\left(E_{\alpha}^{b d}, E_{\delta}^{b d}, E_{\gamma}^{b d}, E_{\beta}^{b d}, E_{\alpha}^{a d}, E_{\delta}^{a d}, E_{\gamma}^{a d}, E_{\beta}^{a d}, d_{E}\right)=0 \\
f_{I}\left(I_{\alpha}^{b d}, I_{\delta}^{b d}, I_{\gamma}^{b d}, I_{\beta}^{b d}, I_{\alpha}^{a d}, I_{\delta}^{a d}, I_{\gamma}^{a d}, I_{\beta}^{a d}, d_{I}\right)=0
\end{gathered}
$$

where $f$ - equations for certain resource-exchange processes (lower index); indicators of resource-exchanges are titled by letters of relevant managerial functions, their lower indexes title subsystems relevant to them ( $\alpha-$ environment, $\beta$ - process, $\delta$ - object, $\gamma$ - project) and upper indexes title periods of time (bd-before decision, $a d$ - after decision); $d$ - parameters of the management decision taken into account, the subscript means the corresponding process of resource exchange.

$$
\left\{\begin{array}{c}
f_{P}\left(P_{\alpha}^{b d}, P_{\delta}^{b d}, P_{\gamma}^{b d}, P_{\beta}^{b d}, P_{\alpha}^{a d}, P_{\delta}^{a d}, P_{\gamma}^{a d}, P_{\beta}^{a d}, d_{A}, d_{E}, d_{I}\right)=0 \\
f_{A}\left(A_{\alpha}^{b d}, A_{\delta}^{b d}, A_{\gamma}^{b d}, A_{\beta}^{b d}, A_{\alpha}^{a d}, A_{\delta}^{a d}, A_{\gamma}^{a d}, A_{\beta}^{a d}, d_{P}, d_{E}, d_{I}\right)=0 \\
f_{E}\left(E_{\alpha}^{b d}, E_{\delta}^{b d}, E_{\gamma}^{b d}, E_{\beta}^{b d}, E_{\alpha}^{a d}, E_{\delta}^{a d}, E_{\gamma}^{a d}, E_{\beta}^{a d}, d_{P}, d_{A}, d_{I}\right)=0 \\
f_{I}\left(I_{\alpha}^{b d}, I_{\delta}^{b d}, I_{\gamma}^{b d}, I_{\beta}^{b d}, I_{\alpha}^{a d}, I_{\delta}^{a d}, I_{\gamma}^{a d}, I_{\beta}^{a d}, d_{P}, d_{A}, d_{E}\right)=0
\end{array}\right.
$$

The detailed structure of these equations is formed on the basis of the following conclusions. Phenomenological models, by their definition, include the relationship between increments that describe the development of the modeled system and its response to external influences. The development of the mega-system shown in Figure $l$ is decomposed into the dynamism of 4 resource exchange processes.

Each one of these processes may be titled abstractly as $X$. Its development is modeled as a pair of curves in Figure 2.

Here the following system of coordinates is used: horizontal coordinate line $\left(X_{\text {long }}\right)$ is for the indicators of $\mathrm{X}$-process in sub-systems with long-distance action (environment and process). Vertical coordinate line $\left(X_{\text {short }}\right)$ is for the indicators in subsystems with short-distance action (object and project). In these coordinates, the two curves express two dependences. The solid curve passing through the entire coordinate plane expresses the dependence of the indicator of the object on the indicator of the environment (since these two subsystems are not limited in time). The dashed curve, which occupies only a part of the plane, is the relationship between the project and the process (both are time-limited).
A managerial decision results in the interaction of two pairs of related subsystems. In the graphical model, this is expressed as the intersection of two curves. Each of these two curves is similar to the trajectory of physical movement. Moreover, at each point it is characterized by speed: from a geometric point of view, it is a tangent to the corner of the curve at this point, and formally it is the ratio between the increments of the dependent and determining indicators.

Making and realizing their decision, a manager corrects increments of the indicators in objectional and projective sub-systems, and this correction is just characterized by parameter $d_{x}$ for certain recourse-exchange. Owing to this correction two curves cross each other - pairs of sub-systems come to interaction.

Figure 2 shows the simplest example, when the indicators of the X-process are known two moments before the decision is made: start - indicators with the superscript $b d . s$., final - with the superscript $b d . f$. They give increments of indicators in final point of period before decision ( $\Delta$-s with upper indicator $b d . f$.). The ratios of these increments are tangent to the angles of the curves at the end point before a decision is made. At the moment of time after the decision is made, the indicators of the $\mathrm{X}$-process in 
subsystems with remote action have increments that are absolutely independent of the manager. While the increments in subsystems with action over short distances have independent parts (the letter $\mathrm{i}$ in brackets in their names) and a $d_{x}$ part, which is the result of a managerial decision. And thanks to this part, the two curves intersect with each other.

The last important question here is how to choose the $d_{x}$ value? The answer to this question, like all other results of this study, is based on the basic principles of the phenomenological approach. They say that the facts (phenomena) from the previous experience of the system under study should not be investigated to determine their root causes. These facts should only be generalized to give the system a way of behaving in the near future. And according to this principle, in the proposed method of making urgent decisions, the phenomenological equation of a single resource exchange process $X$ equalizes the increment relations before and after the managerial decision. Therefore, the parameter $d_{x}$ should be taken into account in such a way as to preserve the equality of the two parts in the formula (3):

$$
\frac{\Delta x(i)_{\delta}^{a d}+d_{x}}{\Delta x_{\alpha}^{a d}}+\frac{\Delta x(i)_{\gamma}^{a d}+d_{x}}{\Delta x_{\beta}^{a d}}=\frac{\Delta x_{\delta}^{b d . f}}{\Delta x_{\alpha}^{b d . f}}+\frac{\Delta x_{\gamma}^{b d . f}}{\Delta x_{\beta}^{b d . f}}
$$

The idea of using the model is as follows: if the modeled system in the previous period has not yet come to collapse with certain ratios between the increments of the resource exchange rates, it means that in the near future approximately the same relations should remain in it. This is a temporary solution made under extreme circumstances for a problem without knowing its specifics. And after the end of the short-term period, a new cognitive process must occur, leading to a new short-term solution or a long-term solution based on a model with a deeper description of the controlled economic system.

Equation (3) has a broader version for the situation when, in the period before the decision is made, there are $n$ number of points at which the indicators of resource exchange are known (4):

$$
\frac{\Delta x(i)_{\delta}^{a d}+d_{x}}{\Delta x_{\alpha}^{a d}}+\frac{\Delta x(i)_{\gamma}^{a d}+d_{x}}{\Delta x_{\beta}^{a d}}=\frac{\sum_{k=2}^{n}\left(\frac{\Delta x_{\delta}^{b d . k}}{\Delta x_{\alpha}^{b d . k}}+\frac{\Delta x_{\gamma}^{b d . k}}{\Delta x_{\beta}^{b d . k}}\right)}{n-1}(4)
$$

And the combination of four such equations through $d$-parameters (as in the mathematical system (2)) gives a general phenomenological model, which is the basis for making urgent decisions for nonstandard problems - this is a mathematical system of equations that connects the increments of resource exchange - indicators (5).

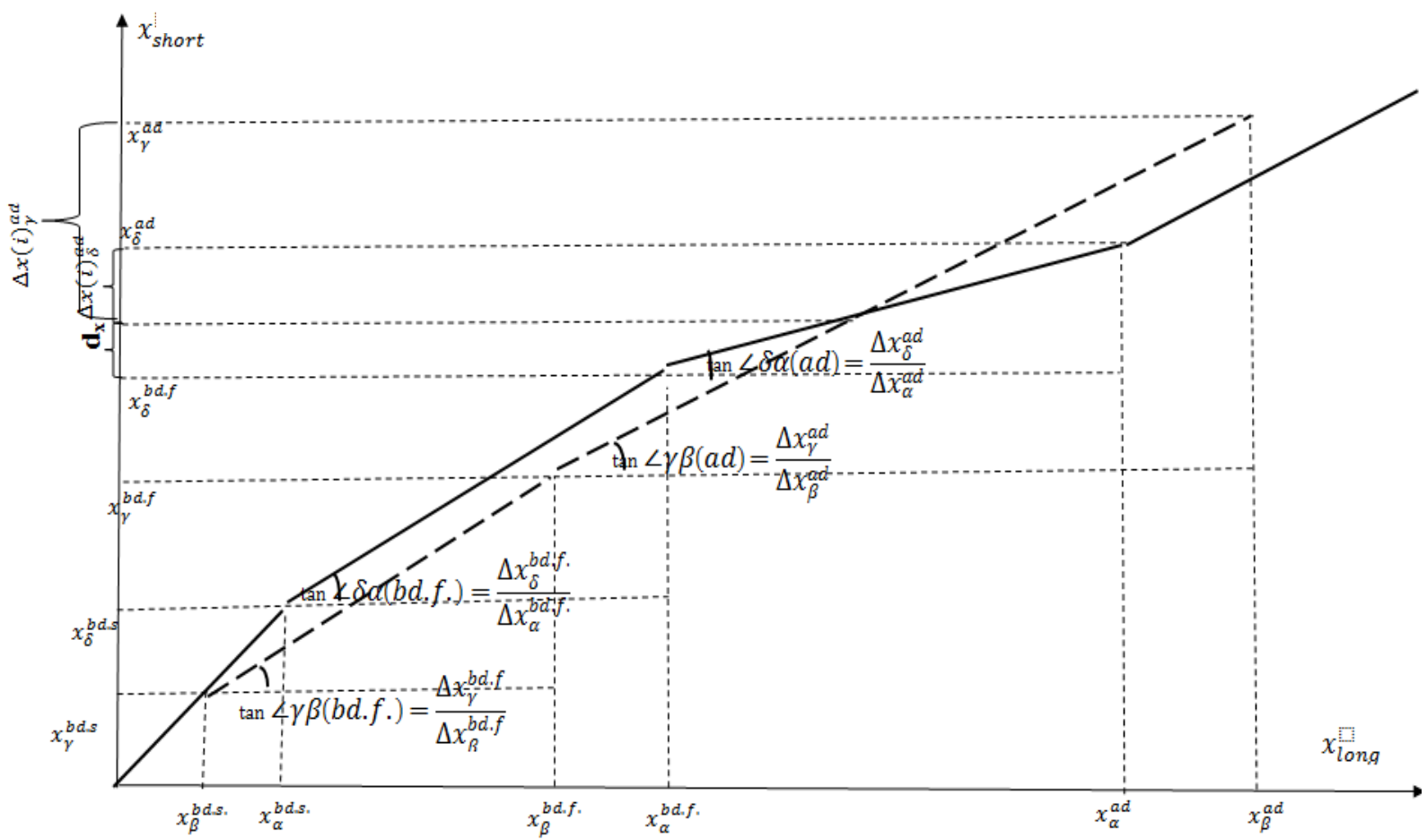

Figure 2. Interaction of sub-systems as a result of managerial decision Source: Compiled by the authors 


$$
\left\{\begin{array}{l}
\frac{\Delta P(i)_{\delta}^{a d}+d_{P}\left(d_{A}, d_{E}, d_{I}\right)}{\Delta P_{\alpha}^{a d}}+\frac{\Delta P(i)_{\gamma}^{a d}+d_{P}\left(d_{A}, d_{E}, d_{I}\right)}{\Delta P_{\beta}^{a d}}=\frac{\sum_{k=2}^{n}\left(\frac{\Delta P_{\delta}^{b d . k}}{\Delta P_{\alpha}^{b d k}}+\frac{\Delta P_{\gamma}^{b d . k}}{\Delta P_{\beta}^{b d k}}\right)}{n-1} \\
\frac{\Delta A(i)_{\delta}^{a d}+d_{A}\left(d_{P}, d_{E}, d_{I}\right)}{\Delta A_{\alpha}^{a d}}+\frac{\Delta A(i)_{\gamma}^{a d}+d_{A}\left(d_{P}, d_{E}, d_{I}\right)}{\Delta A_{\beta}^{a d}}=\frac{\sum_{k=2}^{n}\left(\frac{\Delta A_{\delta}^{b d k}}{\Delta A_{\alpha}^{b d k}}+\frac{\Delta A_{\gamma}^{b d k}}{\Delta A_{\beta}^{b d k}}\right)}{n-1} \\
\frac{\Delta E(i)_{\delta}^{a d}+d_{E}\left(d_{P}, d_{A}, d_{I}\right)}{\Delta E_{\alpha}^{a d}}+\frac{\Delta E(i)_{\gamma}^{a d}+d_{E}\left(d_{P}, d_{A}, d_{I}\right)}{\Delta E_{\beta}^{a d}}=\frac{\sum_{k=2}^{n}\left(\frac{\Delta E_{\delta}^{b d k}}{\Delta E_{\alpha}^{b d k}}+\frac{\Delta E_{\gamma}^{b d k}}{\Delta E_{\beta}^{b d k}}\right)}{n-1} \\
\frac{\Delta I(i)_{\delta}^{a d}+d_{I}\left(d_{P}, d_{A}, d_{E}\right)}{\Delta I_{\alpha}^{a d}}+\frac{\Delta I(i)_{\gamma}^{a d}+d_{I}\left(d_{P}, d_{A}, d_{E}\right)}{\Delta I_{\beta}^{a d}}=\frac{\sum_{k=2}^{n}\left(\frac{\Delta I_{\delta}^{b d k}}{\Delta I_{\alpha}^{b d k}}+\frac{\Delta I_{\gamma}^{b d k}}{\Delta I_{\beta}^{b d k}}\right)}{n-1}
\end{array}\right.
$$

Although these mathematical constructs seem too abstract, in fact they reflect the processes that practically take place when making urgent solutions to non-standard problems. In such situations, the essence of the manager's activity comes down to the integration of two types of information: the first has already been used for previous similar decisions, the second is individual only for the present decision. Their integration ultimately leads the manager to a decision. But in current practice, this is an informal, intuitive process. We propose to systematize it using a phenomenological model of interaction between pairs of subsystems "environment - object" (old information) and "process - project" (new information) with a management decision as the final result.

The proposed methodological principles have already been tested for practical tasks related to the management of the creation and application of innovative oil refining technologies [11].

\section{DISCUSSION}

The main problem with this new methodology is its harmonization with classical decision theory, which insists on choosing the best alternative for a controlled system. However, the conditions of modern economic life (characterized by the new definition of VUCA) in many cases do not give practicing managers the opportunity to use classical decision-making algorithms. The reasons are the growing instability and ignorance of many new situations at work.

So, phenomenological modeling covers the need for methodological tools for management activities in such situations. In addition, the proposed methodology does not deny classical decisionmaking algorithms. It is aimed at strengthening them, and phenomenological models become the basis for models that describe long-term patterns of development of economic systems and form the basis for long-term decisions.

\section{CONSLUSION}

This study has achieved its goal, since the basic methodological principles of algorithmic processes for making urgent decisions for non-standard tasks were created. And these principles are based on phenomenological modeling, the methods of which are adapted to the peculiarities of economic systems as objects of management. The proposed principles have been successfully tested in practice.

The following activities are adopted to further develop these new methodological principles:

- an increase in the number of practical management arenas with successful experience of using phenomenological models to make urgent decisions;

- creation of special software products for the computerized use of these models;

- creation of a methodology for using phenomenological models as the basis for models describing the long-term laws of development of economic systems for making strategic decisions in them.

\section{AUTHORS' CONTRIBUTIONS}

The authors made an equal contribution to the study: collection and analysis of material; definition of goals and objectives, research methods; formulation and scientific substantiation of conclusions, registration of key research results in the form of an article. 


\section{REFERENCES}

[1] A.I. Priven, A.T. Kynin, "A phenomenological model of parameter growth in engineering systems", International Journal of Systematic Innovation, 2012, vol. 2, pp. 9-23. DOI: https://doi.org/10.6977/IJoSI.201209_2(2).0002

[2] L.S. Maergoiz, R.G. Khlebopros, "The indicator of "happiness" in the resource-based economy: an extreme approach", Journal of Siberian Federal University. Humanities and Social Sciences, 2016, vol. 9(8), pp. 1739-1745. DOI: https://doi.org/10.17516/1997-1370-2016-9-81739-1745

[3] I.Y. Tyukin, A.N. Gorban, K.I. Sofeykov, I. Romanenko, "Knowledge transfer between artificial intelligence systems", Frontiers in Neurorobotics, 2018, vol. 12, p. 49. DOI: https://doi.org/10.3389/fnbot.2018.00049

[4] V.K. Semenychev, E.I. Kurkin, A.A. Danilova, E.V. Semenychev, "Multimodel forecasting of non-renewable resources production", Energy, 2017, vol. 130, pp. 448-460. DOI: http://dx.doi.org/10.1016/j.energy.2017.04.098

[5] M.B. Ianenko, L.A. Badalov, Y.A. Rovensky, G.A. Bunich, E.B. Gerasimova, "Essence, risks and control of uncertainties in the process of making investment decisions", Espacios, 2018, vol. 39(31).

[6] I.N. Drogobetsky, "System cybernatization of organizational management" [Sistemnaya kibernetizatsiya organizatsionnogo upravleniya], Moscow: University Book, 2018, 332 p. (In Russ.).

[7] M. Gumerov, "New approaches and models in organizational management under the circumstances of modern economy" [Novyye podkhody i modeli organizatsionnogo upravleniya $\mathrm{v}$ usloviyakh sovremennoy ekonomiki], Moscow: Professor, 2018, 289 p. (In Russ.).

[8] N. Winer, "Cybernetics: or control and communications in the animal and the machine", Paris-Cambridge: Hermann \& CieThe Technology Press, 1948.

[9] G.B. Kleiner, V.A. Karpinskaya, "Transition of firms from the traditional to ecosystem form of business: the factor of transaction costs", Lecture notes in networks and systems, 2020, vol. 110, pp. 3-14. DOI: https://doi.org/10.1007/978-3-030-45913-0_1

[10]I. Adizes, "The Pursuit of Prime", Santa Monica: Knowledge Exchange, 1996.

[11]M. Gumerov, "Methodology of extra decisionmaking in industry on the base of phenomenological models" [Metodologiya formirovaniya ekstrennykh upravlencheskikh resheniy na promyshlennykh predpriyatiyakh $\mathrm{s}$ primeneniyem fenomenologicheskikh modeley], Moscow: Central Economic Mathematical Institute, 2020. (In Russ.). 